\title{
Features of prognostic significance in testicular germ cell tumours
}

\author{
CONSTANCE PARKINSON ${ }^{1}$ AND J. O. W. BEILBY \\ From the Bland Sutton Institute (Gynaecological Pathology), The Middlesex Hospital Medical School, \\ London W1N $8 A A$
}

SUMMARY One hundred and one testicular tumours previously diagnosed as 'teratoma' were examined and 93 of the patients were followed up. These neoplasms were assigned to one of three groups. Those composed exclusively of somatic tissues were the only tumours referred to as teratoma. The second group was exclusively extra embryonic and consisted of either yolk sac or choriocarcinoma. Neoplasms in the third group were called mixed germ cell tumours and incorporated somatic and extra embryonic tissue and occasionally seminoma. The patients with teratomas showed a very low mortality whereas pure yolk sac tumours proved highly malignant. In mixed germ cell tumours the malignant nature of the yolk sac component was maintained even when combined with somatic elements, but when seminoma was also present the survival rate was significantly improved.

It would appear that yolk sac tumour tissue occurs more frequently in adult testicular neoplasms than was previously suspected and in mixed germ cell tumours it can be expected to dictate behaviour except when combined with seminoma.

Within the pleomorphic group of gonadal tumours often referred to as 'teratoma' orthodox methods of histological grading are usually inapplicable. Choriocarcinomatous foci, however, are regarded as prognostically sinister. In a study of ovarian mixed germ cell tumours (solid 'teratoma') we concluded that a tumour pattern, considered to be yolk sac in origin, was an important prognostic guide (Beilby and Parkinson, 1975). Based on these findings and the knowledge that serum alpha-fetoprotein (a product of human yolk sac) (Gitlin and Perricelli, 1970 ) is raised in patients with testicular 'teratocarcinoma' (Norgaard-Pedersen et al., 1975), we investigated the incidence and relation to prognosis of yolk sac tumour in testicular germ cell neoplasms.

\section{Material and methods}

One hundred and one testicular tumours previously reported as 'teratoma' at the Middlesex Hospital between 1923 and 1970 were reviewed. Detailed histories were available in 93 of the 101 patients, all of whom had been treated by surgery and radiotherapy. The survivors were followed up for three 'Present address: Department of Forensic Medicine, Guy's Hospital Medical School, London SE1 9RT

Address for reprints: J. $O$. W. Beilby, The Bland-Sutton Institute, The Middlesex Hospital Medical School, London W1N 8AA

Received for publication 8 June 1976 to 37 years. Paraffin wax blocks from each primary neoplasm were sectioned and stained with haematoxylin and eosin. The tumours, with the exception of one which was undifferentiated, were assigned to one of the following three groups, and the clinical behaviour of these was compared: 1 teratoma, ie, those neoplasms composed exclusively of mature or immature somatic elements; 2 tumours composed exclusively of extra embryonic tissue, ie, choriocarcinoma or yolk sac tumour. The latter was identified by its characteristic range of merging patterns, including Duval-Schiller bodies, intercommunicating channels lined by flattened epithelium, clear celled reticular and microcystic formations, and acinopapillary structures lined by cuboidal and columnar epithelium (Figs. 1-4); 3 mixed germ cell tumours composed of both types of tissues listed in 1 and 2 , and various combinations of these with seminoma (Figs. 5 and 6). In this group an approximate quantitation of the proportion of yolk sac tissue was made by a scoring system which allowed each neoplasm to be graded + to +++ .

All tumours were also classified according to Pugh (1976) and Mostofi and Price (1973).

Metastases were examined in six cases from our series. In addition, secondary deposits from 52 other patients with testicular 'teratoma' were reviewed. 


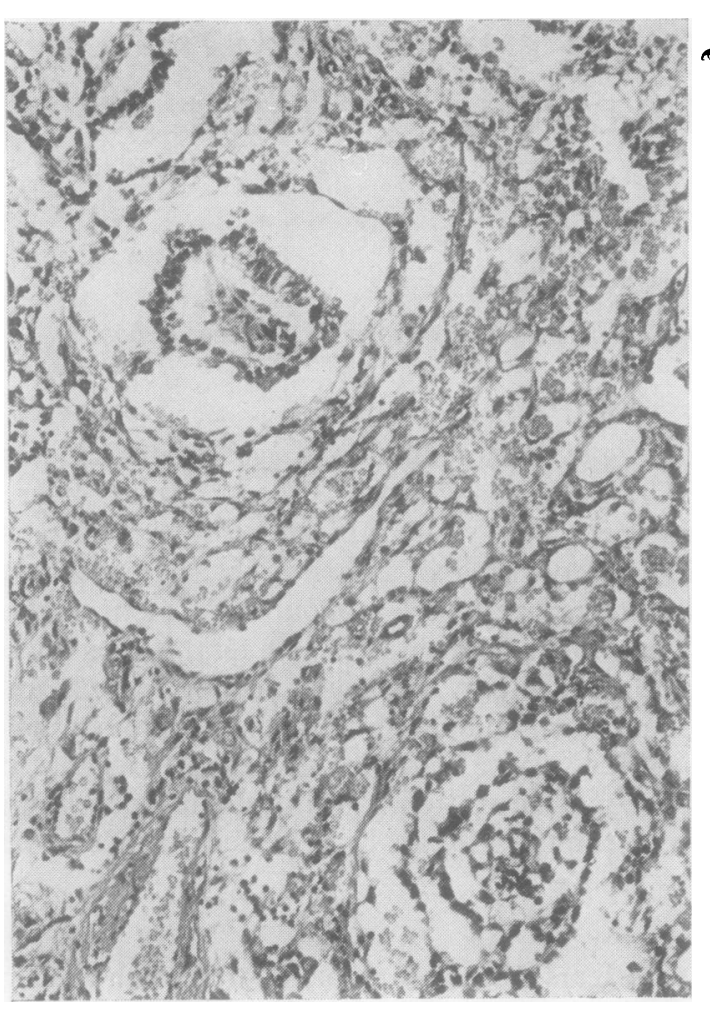

Fig. 1 Yolk sac pattern including two Duval-Schiller bodies (Haematoxylin and eosin $\times 132$ ).

\section{Results}

The ages of the 92 patients whose testicular tumours were admissible for classification ranged from 3 months to 52 years (Table). Only four were under 10 years old, the highest incidence of tumour being in the third decade. In group I (teratoma) six of the seven patients survived. Thirty-four tumours were extra embryonic in type (group 2), 33 of which were yolk sac and one was a choriocarcinoma. Eight of the patients with yolk sac tumour were alive and well; the solitary choriocarcinoma proved fatal. The remaining 51 neoplasms were assigned to the mixed germ cell group. Thirty-three of these consisted of yolk sac and somatic elements; nine of the patients survived. Fourteen mixed germ cell tumours incorporated yolk sac and seminoma, somatic elements being present in eight of these; 11 patients were alive and well. The improved survival rate in patients whose mixed germ cell tumours contained seminoma in addition to yolk sac tumour was statistically significant $(\mathrm{P}<0.001)$. The one patient whose neoplasm was composed of somatic elements and seminoma survived. The three mixed germ cell $\stackrel{0}{5}$ tumours containing choriocarcinoma proved fatal.

In the 33 mixed germ cell neoplasms composed $\underset{\vec{B}}{\vec{B}}$ of somatic elements and yolk sac tumour, the $\frac{9}{\circ}$ following association was found between survival $\frac{\square}{\circ}$ and the proportion of yolk sac tumour present. $\overline{\bar{N}}$ Four out of 10 patients with neoplasms graded,$+ \widehat{\nabla}$ four out of 18 graded ++ , and one out of five $\varrho$ graded +++ survived. This quantitative relation- $\infty$ ship was not apparent in the presence of seminoma. $\overrightarrow{0}$

The six metastases in our series consisted exclu- $\overrightarrow{-}$ sively of yolk sac tumour (Fig. 7). Two of the primary $\vec{\sigma}$ neoplasms were yolk sac tumours, the others were mixed germ cell tumours incorporating yolk sac? elements, with somatic components in three instances and seminoma in one. Among the further ? 52 secondary deposits examined, 21 were composed $\vec{\omega}$ exclusively of yolk sac tumour, 11 of chorio- 0 carcinoma, and five were a mixture of the two. $]$ Somatic elements were seen in only three cases, in 7 one instance as the sole metastatic component and $\mathbb{D}$ in the other two cases associated with yolk sac tumour. Two secondary deposits were undifferentiated and 10 proved too necrotic to justify an opinion. $\vec{\varphi}$

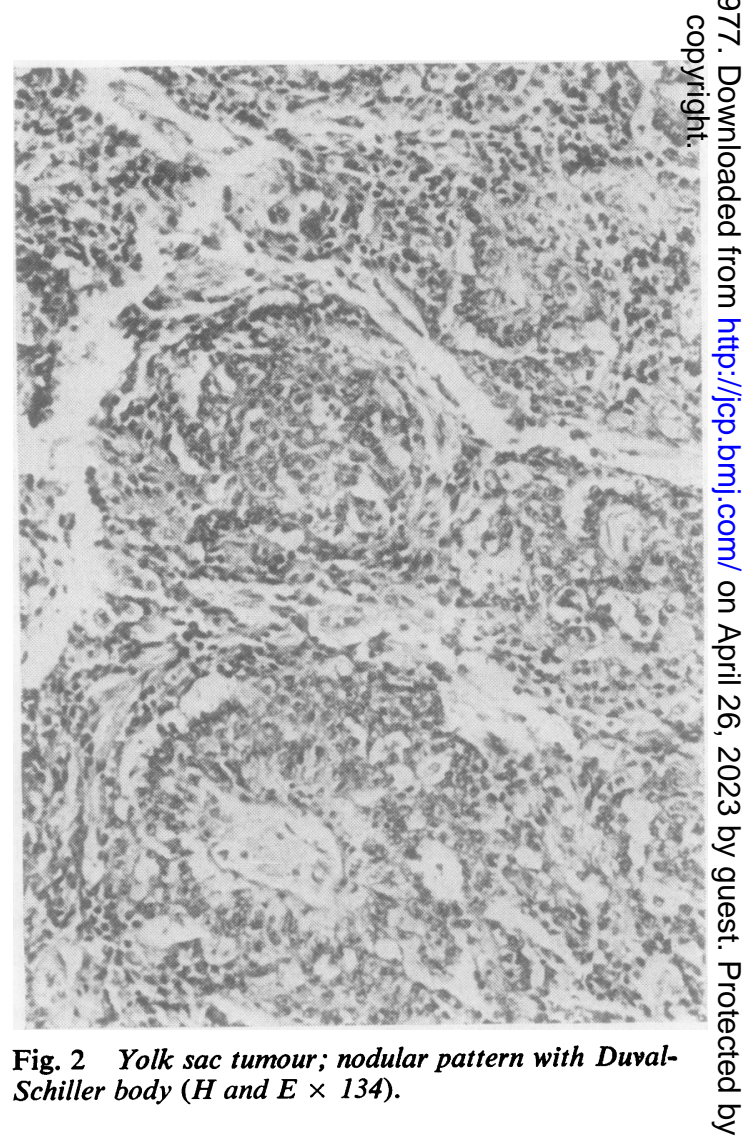




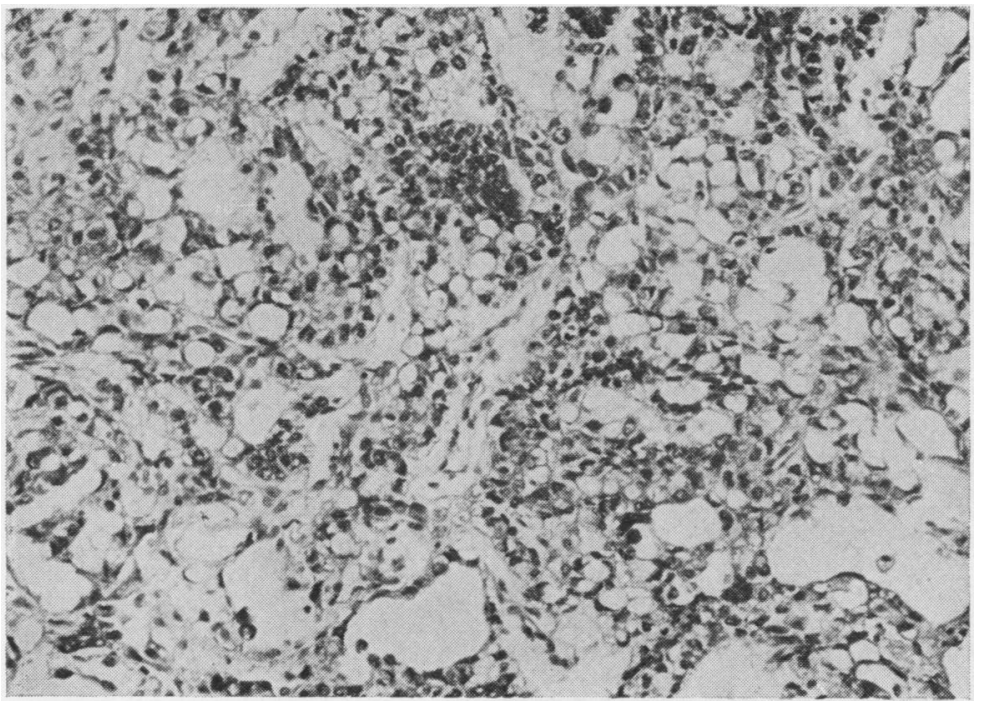

Fig. 3 Yolk sac tumour, reticular and microcystic pattern ( $H$ and $E \times 150)$.

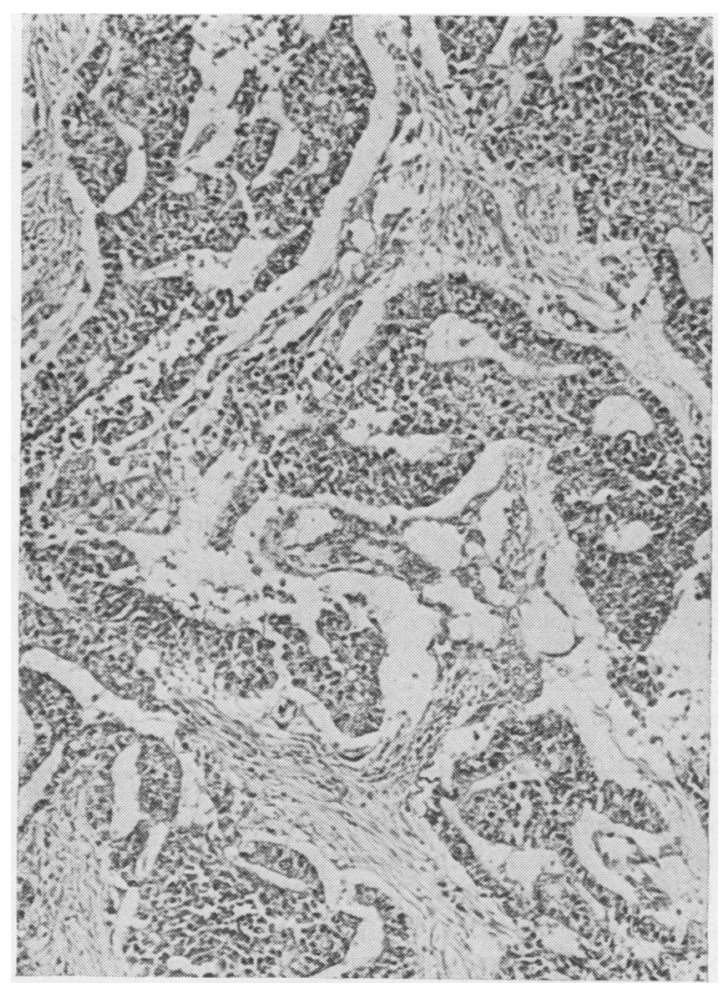

Fig. 4 Yolk sac tumour; acinopapillary pattern. The epithelial appearance is consistent with a visceral yolk sac origin ( $H$ and $E \times 68)$.

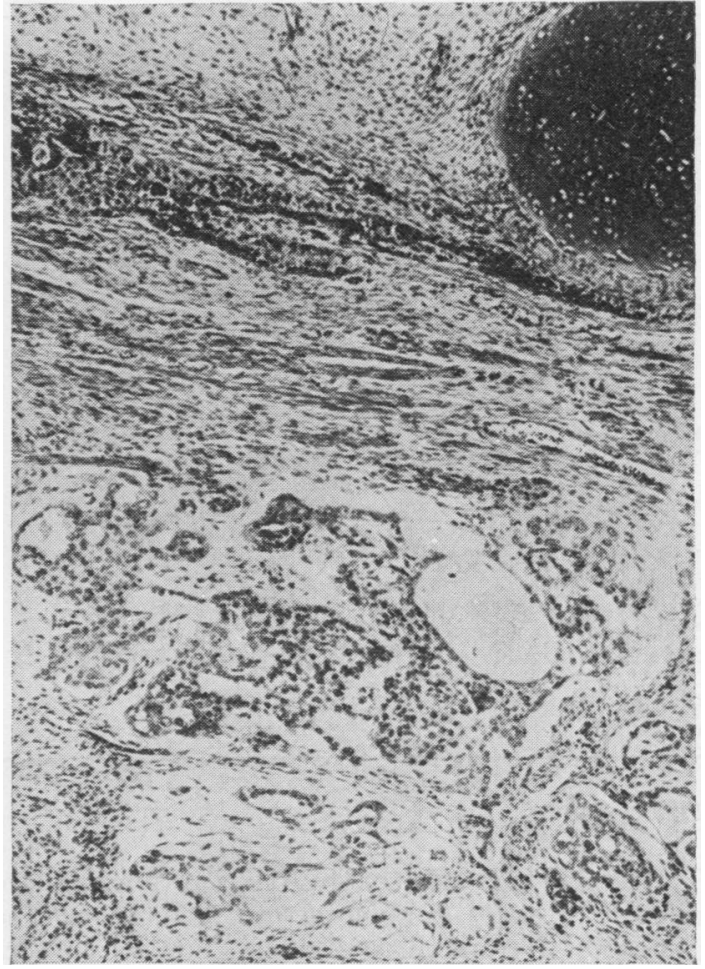

Fig. 5 Primary mixed germ-cell tumour showing both somatic tissues and a yolk sac component in the lower half of the picture $(H$ and $E \times 71)$. 


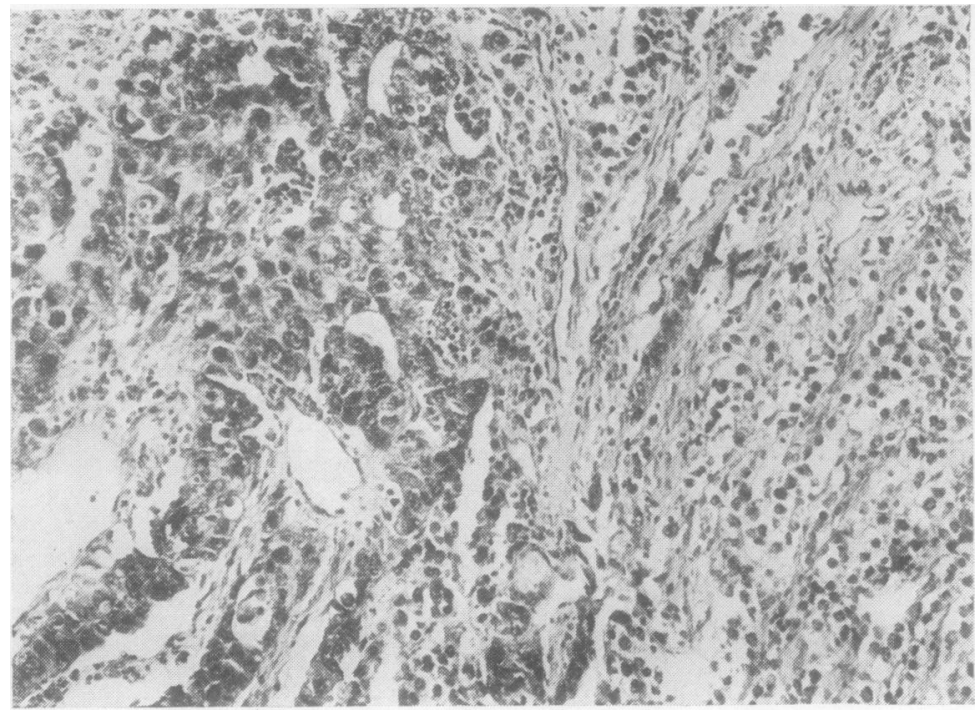

Fig. 6 Primary mixed germ cell tumour with seminomatous and yolk sac components ( $H$ and $E \times 144)$.

Table Relationship of histological pattern to survival in 92 germ cell tumours of the testis

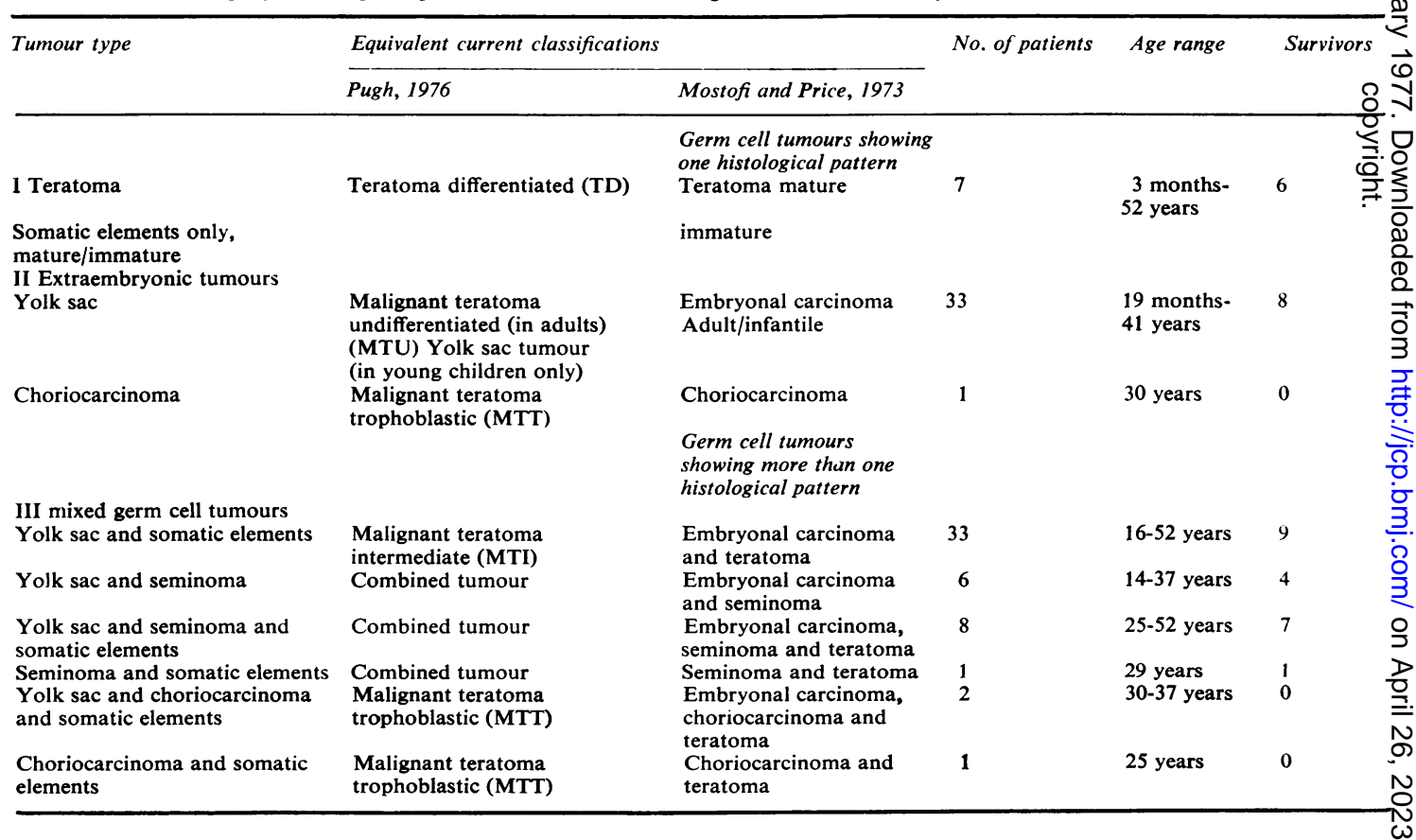

\section{Discussion}

Traditional methods of histological grading are difficult to apply to some germ cell tumours. The presence of trophoblastic tissue, however, either pure or mixed with other germ cell elements, is universally regarded as a sinister prognostic feature. In the ovary, an extra embryonic tumour of yolk sac origin occurs in either pure form (Beilby and Todd 1974) or mixed with other germ cell componentso (Beilby and Parkinson, 1975). The range of morphological patterns and the malignant nature of this ${ }^{\infty}$ ovarian neoplasm is accepted (Teilum, 1971) while the significance of the same histological appearances? in testicular tumours is obscured by synonyms and? different methods of classification. More recently@ 


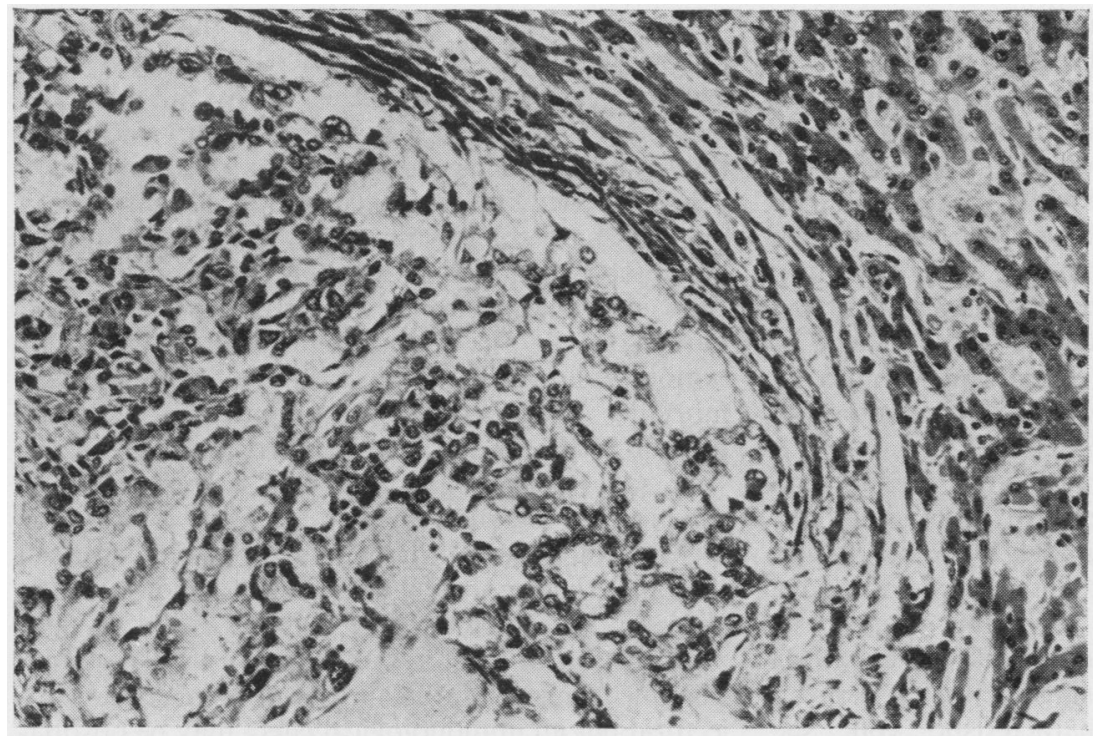

Fig. 7 Pure yolk sac metastasis (reticular pattern) in liver from the primary mixed germ cell tumour shown in Fig. 5 ( $H$ and $E \times 170)$.

reports recognising adult testicular yolk sac tumour have been published (Talerman, 1974 and 1975). We submit that distinctive adenocarcinoma of the infant testis (Magner et al., 1956), orchioblastoma (Teoh et al., 1960), some examples of embryonal carcinoma (Dixon and Moore, 1952; Mostofi and Price, 1973), endodermal sinus tumour (Teilum, 1971), and yolk sac tumour (Pierce et al., 1970; Beilby and Todd, 1974) have the same histological patterns. Biochemical (alpha-fetoprotein production) Masopust et al., 1968; Tsuchida et al., 1973; Norgaard-Pedersen et al., 1975), embryological (Marin-Padilla, 1968; Gaillard, 1972), and comparative anatomical (Pierce et al., 1970) evidence supports the contention that these neoplasms have a yolk sac origin. The general acceptance of this concept will enable the true incidence, age range, and clinical behaviour of testicular yolk sac tumour to be documented and the importance of alpha-fetoprotein levels in such patients to be assessed.

The terms distinctive adenocarcinoma of the infant testis and orchioblastoma, while specifying a distinctive tumour pattern, restrict its occurrence to male infants. The dissociation of these testicular tumours from those of similar pattern in the adult ovary is justified by the better prognosis reported in younger males. This discrepancy is not a consistent finding and may be a consequence of earlier detection and treatment, as is evident in the series of Pierce et al. (1970), rather than an inherent quality of the tumour. The use of the above two terms and the contention that the yolk sac tumour of the testis occurs only in infants (Pugh, 1976a) tends to eliminate appreciation of the true age range and prognostic significance of yolk sac tumour in the adult male. In the classification of testicular tumours currently used in Britain (Pugh, 1976b), the importance of choriocarcinoma in dictating a poor prognosis is acknowledged in the group 'malignant teratoma trophoblastic' (MTT), while the morphological appearances and significance of a second extra embryonic yolk sac component in the adult goes unrecognised. Considerable emphasis is laid on organoid somatic elements whose presence denotes maturation of the tumour as a whole and indicates a more favourable prognosis ('malignant teratoma intermediate' (MTI)) than for those neoplasms where somatic elements are absent ('malignant teratoma undifferentiated' MTU)). We acknowledge that a neoplasm giving rise to both somatic and extra embryonic tissue may exist, but in practice we find that totally undifferentiated tumours composed of sheets of primitive cells are rare. In MTI and MTU, tissue referred to as 'undifferentiated' frequently shows yolk sac morphology from which it is unlikely that other germ cell elements develop. Thus we find the terms mixed germ cell tumour and yolk sac tumour more accurate descriptions for most cases in the groups MTI and MTU respectively. When we apply the current British classification (Pugh, 1976) to our series we fail to show any prognostic difference between the MTI and MTU groups. We would interpret these findings as showing the malignant nature of yolk sac tumour which is unaltered by the presence of somatic elements. 
Thus we would grade such neoplasms according to the most malignant element rather than the most orientated somatic tissues present. However, there is some indication within the group MTI (somatic elements plus yolk sac tumour) that survival may be related to the quantity of yolk sac tumour. The neoplasms designated 'combined tumours' (Pugh, 1976b) ('teratoma plus seminoma') are found to incorporate yolk sac elements in a high proportion of cases. Statistically we find that the presence of seminoma in such tumours significantly improved the prognosis $(\mathrm{P}<0.001)$, an observation also made previously (Pugh, 1976c).

Despite its epithelial connotations, Dixon and Moore (1952) favoured the term embryonal carcinoma for the totipotent tumour inherent in their pathogenic concept of teratoma. We acknowledge the experimental evidence for this hypothesis (Pierce and Dixon, 1959; Pierce and Verney, 1961) but question the logic of basing such a concept on tumour tissue showing characteristic yolk sac differentiation. Our objection is supported by the tendency for such tumour tissue to retain its characteristic yolk sac pattern at its secondary site rather than differentiating along other germ cell lines. Prognostically we agree with Mostofi and Price (1973) on the importance of identifying and recording each component of a testicular germ cell tumour. Contrary to their observations, we find that the inclusion of seminoma with yolk sac tumour significantly improves prognosis, whereas the presence of somatic elements has no such modifying effect.

It is clear that the above classifications (Mostofi and Price, 1973; Pugh, 1976b) fail to recognise that the range of patterns we have described as yolk sac tumour are expressions of a single neoplastic entity and thus are unsuitable for assessing the incidence and prognostic significance of this extra embryonic tumour. Based on our contention that yolk sac tumour is a significant prognostic marker, we divided the neoplasms in our series into three groups. Tumours composed exclusively of somatic elements carry a good prognosis. The low metastatic potential of somatic components is underlined by their rare occurrence in secondary deposits; they were seen only in three out of 58 metastases examined, despite their recognised frequency in primary testicular mixed germ cell tumours. These observations parallel the findings in homologous ovarian neoplasms (Beilby and Parkinson, 1975).

The second group of tumours is exclusively extra embryonic, predominantly of yolk sac type. The prognosis for patients with such neoplasms is poor, in marked contrast to that of neoplasms composed entirely of somatic elements.
In mixed germ cell tumours, the malignant $\stackrel{\overrightarrow{5}}{\circ}$ nature of the yolk sac element is maintained when $\div$ associated with somatic tissues, the overall mortality $\overrightarrow{\vec{F}}$ resulting from these neoplasms being similar to that of pure yolk sac tumours. Moreover, secondary deposits from known mixed germ cell tumours in $\overline{\bar{c}}$. our series are solely yolk sac in type. These results $\vec{\sigma}$ are comparable with those obtained when homolo- $~$ gous ovarian neoplasms were studied (Beilby and कै Parkinson, 1975). Within this group of testicular $\vec{\circ}$

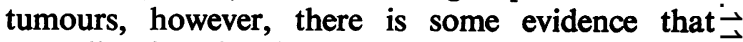
mortality is related to the quantity of yolk sac. present in the primary tumour. In the cases where yolk sac elements are combined with seminoma, prognosis is significantly improved. In view of the $\omega$ high mortality seen in patients with mixed germ cell? tumours incorporating yolk sac and somatic $\vec{\omega}$ elements, we interpret these findings as indicating $a_{\circ}$ modification of yolk sac malignancy by seminoma. The mechanism of this interaction must be con- $T$ jectural but an immunological effect may be postula- $\frac{\mathbb{O}}{0}$ ted in view of the classical lymphocytic infiltrate in seminoma and excellent response to therapy.

Current biochemical experience in demonstrating $\vec{c}$ alpha-fetoprotein in yolk sac tumour tissue (Norgaard- $y$ Pedersen, 1975) and the frequency with which it hiss. been detected in the serum of a larger series of paf ients with testicular 'teratoma' (Kohn et al., personal communication) tends to support our morphological opinion on the incidence and prognosis of yolk sac $\frac{\otimes}{\not}$

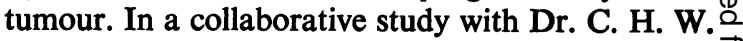
Horne utilising an enzyme bridge immunoperoxidase $\overrightarrow{\vec{O}}$ technique, we are currently investigating the 3 presence of alpha-fetoprotein in tumours of our series. Preliminary results demonstrate alphafetoprotein in the morphological variants of yolk sac tumour we have described.

We thank Professor A. C. Thackray for allowing us access to some of the histological material and $\frac{O}{3}$ Dr R. A. B. Drury for helpful advice. We are also indebted to Miss Joyce Hubbard for photography and to Mrs Elizabeth Mills for typing the manu- $\frac{D}{0}$ script.

\section{References}

Beilby, J. O. W., and Parkinson, C. (1975). Features of prognostic significance in solid ovarian teratoma. Cancer, 36, 2147-2154.

Beilby, J. O. W., and Todd, P. J. (1974). Yolk sac tumour of the ovary. Journal of Obstetrics and Gynaecology of the $\bar{\Phi}$ British Commonwealth, 81, 90-94.

Dixon, F. J., and Moore, R. A. (1952). Germinal tumors. In Tumors of the Male Sex Organs (Atlas of Tumor Pathology, Section 8, Fascicles $31 \mathrm{~b}$ and 32), pp. 55-104. $\frac{+}{\mathbb{D}}$ Armed Forces Institute of Pathology: Washington, DC. Gaillard, J. A. (1972). Yolk sac tumour patterns and ento-苂 blastic structures in polyembryomas. Acta Pathologica 
Microbiologica Scandinavica, Sect. A. 80, Supplement 233, 18-25.

Gitlin, D., and Perricelli, A. (1970). Synthesis of serum albumin, prealbumin, alpha-foetoprotein, $\alpha$-antitrypsin and transferrin by the human yolk sac. Nature, 228, 995-997.

Magner, D., Campbell, J. S., and Wiglesworth, F. W. (1956). Testicular adenocarcinoma with clear cells, occurring in infancy. Cancer, 9, 165-175.

Marin-Padilla, M. (1968). Histopathology of the embryonal carcinoma of the testes: embryological evaluation. Archives of Patkology, 85, 614-622.

Masopust, J., Kithier, K., Rádl, J., Koutecký, J., and Kotál, L. (1968). Occurrence of fetoprotein in patients with neoplasms and non-neoplastic diseases. International Journal of Cancer, 3, 364-373.

Mostofi, F. K., and Price, E. B., Jr. (1973). Tumours of germ cell origin. In Tumors of the Male Genital System. (Atlas of Tumor Pathology, edited by H. I. Firminger, Second series, Fascicle 8), pp. 7-84. Armed Forces Institute of Pathology: Washington, DC.

Norgaard-Pedersen, B., Albrechtsen, R., and Teilum, G. (1975). Serum alpha-foetoprotein as a marker for endodermal sinus tumour (yolk sac tumour) or a vitelline component of 'teratocarcinoma'. Acta Pathologica Microbiologica Scandinavica, Section A., 83, 573-589.
Pierce, G. B., Bullock, W. K., and Huntington, R. W., Jr (1970). Yolk sac tumors of the testis. Cancer, 25, 644-658.

Pierce, G. B., and Dixon, F. J., Jr. (1959). Testicular teratomas, 1 . Demonstration of teratogenesis by metamorphosis of multipotential cells. Cancer, 12, 573-583.

Pierce, G. B., Jr., and Verney, E. L. (1961). An in vitro and in vivo study of differentiation in teratocarcinomas. Cancer, 14, 1017-1029.

Pugh, R. C. B. (1976). Pathology of the Testis, 2nd edition (a) p. 357 , (b) p. 205, (c) p. 255: Blackwell, Oxford.

Talerman, A. (1974). Yolk sac tumor associated with seminoma of the testis in adults. Cancer, 33, 1468-1473.

Talerman, A. (1975). The incidence of yok sac tumor (endodermal sinus tumor) elements in germ cell tumors of the testis in adults. Cancer, 36, 211-215.

Teilum, G. (1971). Special Tumors of Ovary and Testis, p. 144. Munksgaard, Copenhagen: Lippincott, Philadelphia.

Teoh, T. B., Steward, J. K., and Willis, R. A. (1960). The distinctive adenocarcinoma of the infant's testis: an account of 15 cases. Journal of Pathology and Bacteriology, 80, 147-156.

Tsuchida, Y., Saito, S., Ishida, M., Ohmi, K., Urano, Y., Endo, Y., and Oda, T. (1973). Yolk sac tumor (endodermal sinus tumor) and alpha-feto-protein: a report of three cases. Cancer, 32, 917-921. 